\title{
A three phase 3-level inverter with reduced THD factor
}

\author{
J.Iwaszkiewicz ${ }^{1}$ and A.Muc ${ }^{1}$ \\ ${ }^{1}$ Department of Electrical Engineering \\ Gdynia Maritime University \\ 81-87 Morska St., 81-225 Gdynia (Poland) \\ Phone/Fax number:+48 58-5586-471, e-mail: j.iwaszkiewicz@we.am.gdynia.pl, a.muc@we.am.gdynia.pl
}

\begin{abstract}
The paper presents an mathematical analysis of a stepped function denoted as $f_{N=6}$. This function describes precisely an output voltage waveform of a 3-level inverter. The analysis proves that cascaded inverters give a very good performance of the output voltage according to a criterion of minimal THD. Optimal parameters are indicated in the paper. They permit to diminish the THD factor to a level below $21 \%$ while in standard NPC inverters such a result requires special treatment.
\end{abstract}

\section{Key words}

THD factor, cascaded inverters, NPC inverters.

\section{Introduction}

In order to enlarge output power and simultaneously improve shape wave forms generated by inverters it is possible to use multilevel inverters. Among them the NPC3-level inverter is largely used and applied in industry and its properties or drawbacks are well known. But it is possible to design3-level inverters as a composition of two standard 2-level inverters. There are more than one topology solutions permitting to obtain such a result. Most of them have been largely discussed in power electronics literature. They prove that higher level of output power as well as wave form improvement can be reached in many ways.

The simplest idea to have satisfactory results has been illustrated in Fig.1. The circuit presents a one phase 3-level inverter consisting of two HB inverters: $\boldsymbol{F} 1(\boldsymbol{T} 1, \boldsymbol{T} 2, \boldsymbol{T} 3$, T 4$), F 2(T 5, T 6, T 7, T 8)$ and a summing block - $\sum$. The symbol $Z$ represents load. The inverters are supplied by DC sources $U_{D 1}$ and $U_{D 2}$ respectively. Generally the source voltages $U_{D 1}$ and $U_{D 2}$ must not be equal

The simplest way of control consists in simultaneously switching pairs of transistors e.g. T 1-T 4, T $2-T$ 3 , T 5$T \boldsymbol{8}$ and $\boldsymbol{T} \mathbf{6}-\boldsymbol{T} 7$. If transistor drivers generate control signals in the shape of rectangular waves than the cascaded inverter produces a stepped output voltage.

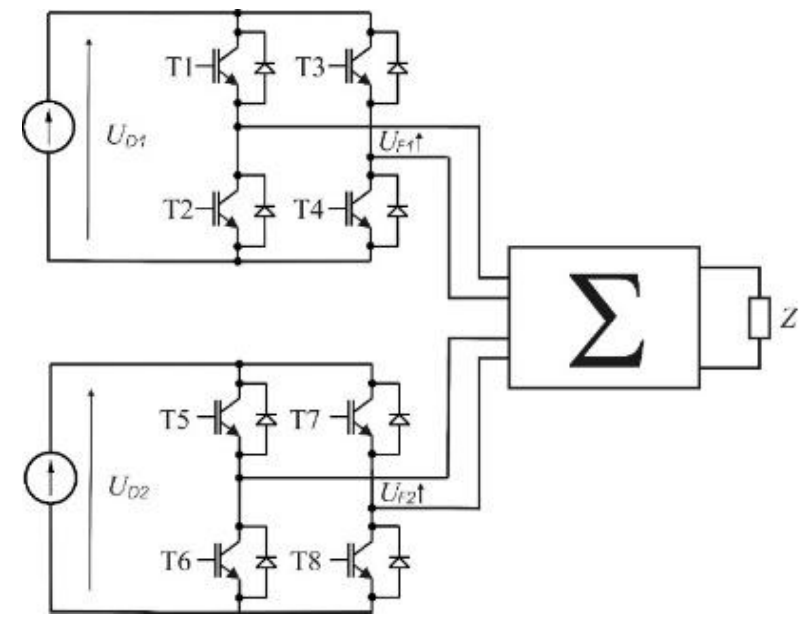

Fig.1. The idea of the one phase cascaded inverter.

The following switching sequence: $T \mathbf{1 - T 4}$, T 1 - T $4 \cap T$ 5-T 8, T 1 - T 4, T 2 -T 3, T 2 - T $3 \cap T 6$ $\boldsymbol{T} 7, \boldsymbol{T} 2-\boldsymbol{T} 3$, where transistor pairs are switched successively on angle range $\pi / 3$ of the period, effects the output voltage takes a form of a stepped function presented in Fig.2. The presented wave form has been obtained when supply voltages $U_{D 1}$ and $U_{D 2}$ were equal $(200 \mathrm{~V})$.In the paper this stepped function will be denoted as $f_{N=6}$ cause it has 6 steps in one period.

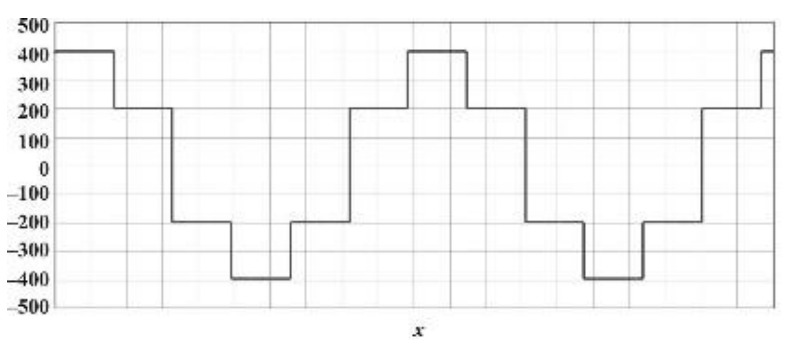

Fig.2. The $f_{N=6}$ output voltage wave form of the cascaded inverter.

In standard NPC 3-level inverters it is possible to obtain the same voltage wave form. The control sequence 
should be different because one phase branch of the NPC inverter has only 4 transistors. The NPC inverter is presented in Fig 3

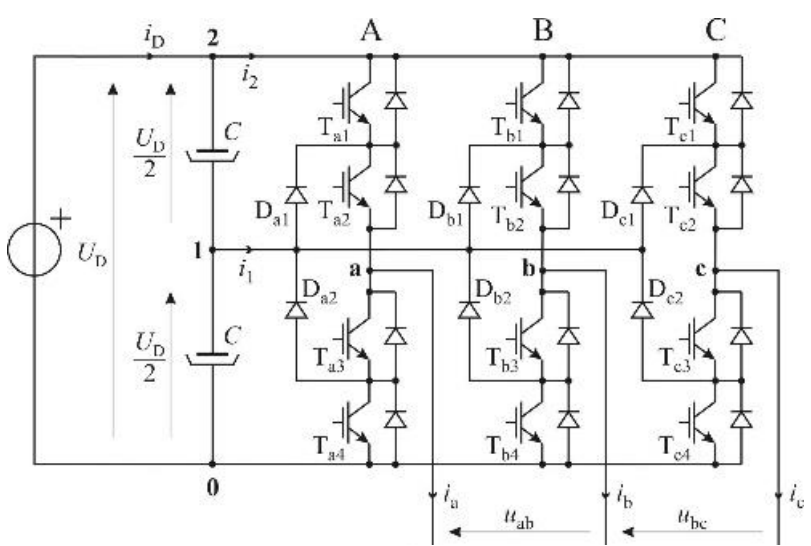

Fig.3. The circuit diagram of the Neutral Point Clamped Inverter.

The following switching sequence in phase A: $\boldsymbol{T} 3 \mathbf{- T} \mathbf{4}$, T 2 - T 3, T 1 - T 2, T 1 - T 2, T 2 - T 3, T 3 - T 4 permits to obtain a stepped voltage wave form. It is assumed that the angle range of each step is $\boldsymbol{\alpha}=\boldsymbol{\pi} / 3\left(\mathbf{6 0}^{\circ}\right)$. In such a case Fig. 4 presents three phase polar voltage wave forms and resulting in a shape of the phase-to-phase voltage $\boldsymbol{u}_{\boldsymbol{A} B \boldsymbol{B}}$. The phase voltages have been referenced to the negative pole of the DC voltage. The negative pole is denoted as $\boldsymbol{0}$ while the positive one as $\mathbf{2}$. Additionally the midpoint potential is denoted as 1 .

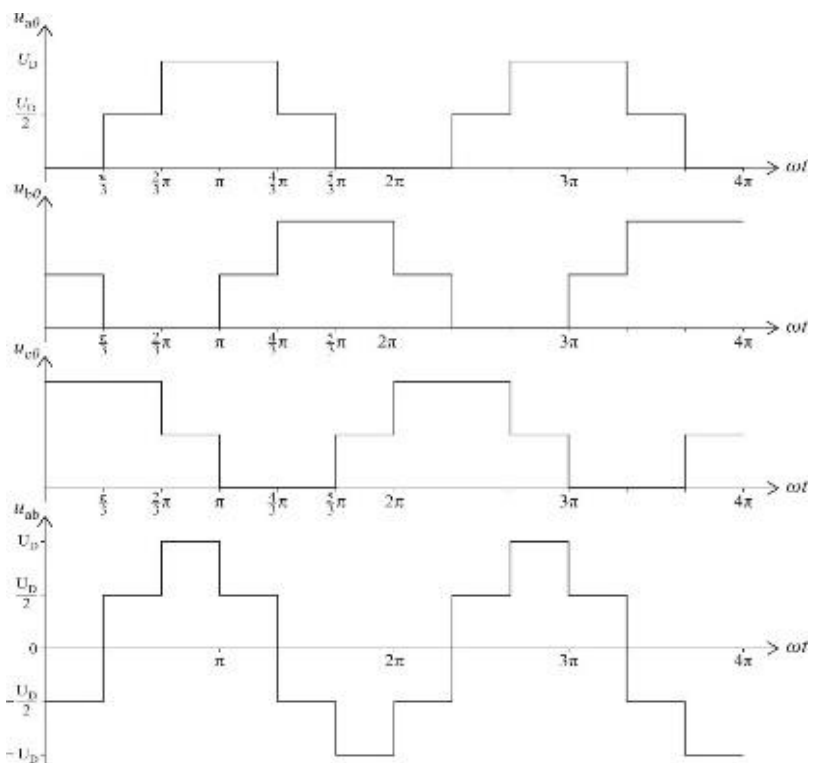

Fig. 4. Phase polar voltage wave forms and phase-to-phase voltage $\boldsymbol{u}_{\boldsymbol{A} \boldsymbol{B}}$.

The phase-to-phase voltage wave form $\boldsymbol{u}_{\boldsymbol{A}}$ takes a form of the $f_{N=6}$ stepped function and may be recognized as a weak approximation of a function $\boldsymbol{f}(\boldsymbol{x})=\sin (\boldsymbol{x})$ in the interval $<\mathbf{0}, \mathbf{2 \pi})$. Fourier transform of the $\boldsymbol{f}_{N=\mathbf{6}}$ stepped function gives a set of harmonics. Assuming the fundamental harmonic equal to 1 it is possible to count all step measures of the $f_{N=6}$ function. The set of coefficients has been presented in Table 1. In order to compare $f_{N=6}$ and $\boldsymbol{f}_{N=\mathbf{1 2}}$ functions the coefficients of the $f_{N=12}$ has been also introduced into Table 1.The $f_{N=6}$ and $f_{N=12}$ function wave forms and their spectra have been presented in Fig. 5 and Fig. 6.

TABLE I. - The coefficients $f_{N}(x)$ for two step numbers $N$ : $N=6, N=12$.

\begin{tabular}{|c|c|c|c|}
\hline Angle range & $\left.<0,60^{\circ}\right)$ & $\left.<60^{\circ}, 120^{\circ}\right)$ & $\left.<120^{\circ}, 180^{\circ}\right)$ \\
\hline$\overline{F_{N}=6}$ & 0,4775 & 0,9549 & 0,4775 \\
\hline Angle range & $\left.<0,30^{\circ}\right)$ & $\left.<30^{\circ}, 60^{\circ}\right)$ & $\left.<60^{\circ}, 90^{\circ}\right)$ \\
\hline$F_{\mathrm{N}=12}$ & 0,2559 & 0,6990 & 0,9549 \\
\hline Angle range & $\left.<180^{\circ}, 210^{\circ}\right)$ & $\left.<210^{\circ}, 240^{\circ}\right)$ & $\left(<240^{\circ}, 270^{\circ}\right)$ \\
\hline$F_{\mathrm{N}=12}$ & $-0,2559$ & $-0,6990$ & $-0,9549$ \\
\hline Angle range & $\left.<180^{\circ}, 240^{\circ}\right)$ & $\left.<240^{\circ}, 300^{\circ}\right)$ & $\left.<300^{\circ}, 360^{\circ}\right)$ \\
\hline$F_{N=6}$ & $-0,4775$ & $-0,9549$ & $-0,4775$ \\
\hline Angle range & $\left.<90^{\circ}, 120^{\circ}\right)$ & $\left.<120^{\circ}, 150^{\circ}\right)$ & $\left.<150^{\circ}, 180^{\circ}\right)$ \\
\hline $\mathbf{F}_{\mathrm{N}=12}$ & 0,9549 & 0,6990 & 0,2559 \\
\hline Angle range & $\left.<270^{\circ}, 300^{\circ}\right)$ & $\left.<300^{\circ}, 330^{\circ}\right)$ & $\left.<330^{\circ}, 360^{\circ}\right)$ \\
\hline$F_{\mathrm{N}=12}$ & $-0,9549$ & $-0,6990$ & $-0,2559$ \\
\hline
\end{tabular}

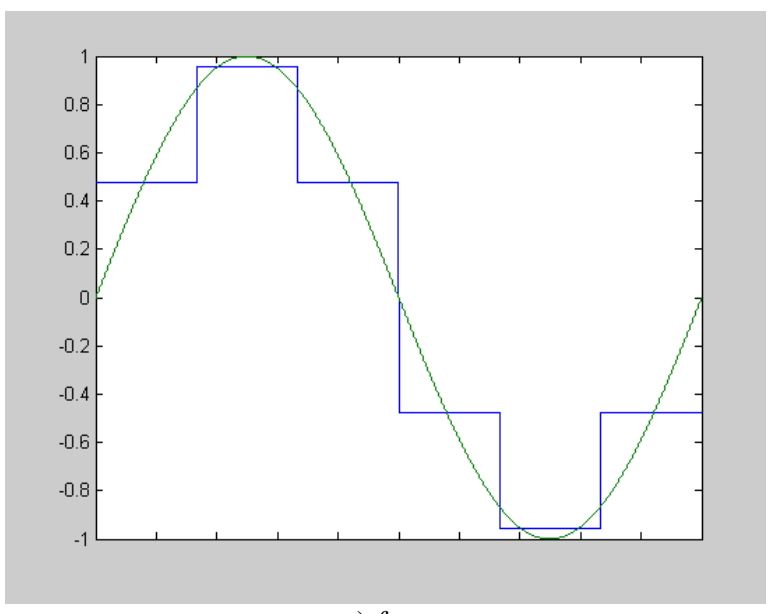

a) $f_{N=6}$

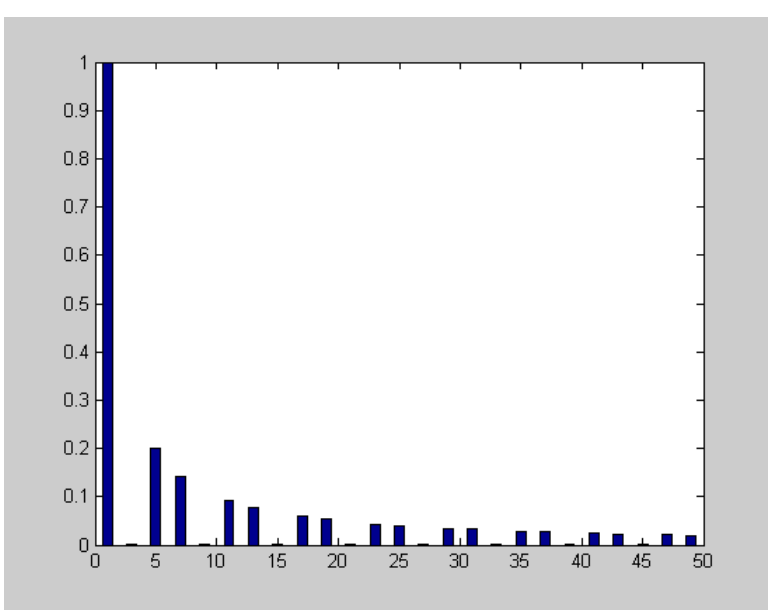

b) $T H D=31,09 \%$

Fig. 5. Stepped function $f_{N=6}$ in the interval $<0,2 \pi$ ): a) wave form $f_{N=6}$, b) spectrum and THD factor. 


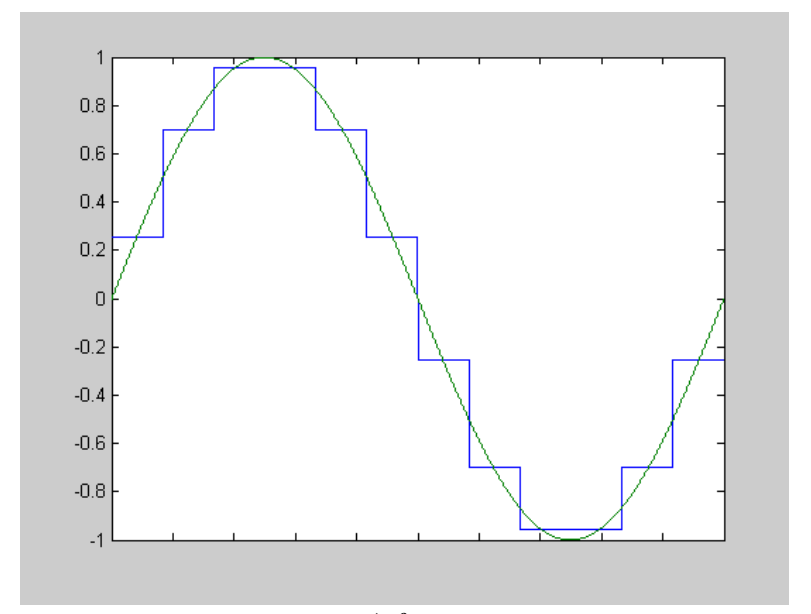

a) $f_{N=6}$

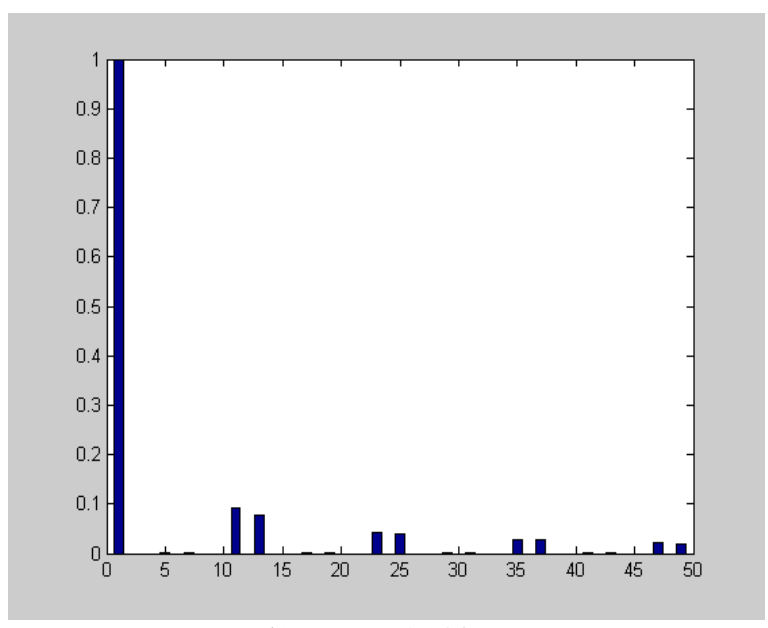

b) $T H D=15,23 \%$

Fig. 6. Stepped function $f_{N=12}$ in the interval $<0,2 \pi$ ): a) wave form, b) spectrum and THD factor.

The most important and effective criterion of approximation quality is the THD (Total Harmonic Distortion) factor. Hence in Fig. 5 and Fig. 6 THD factors of spectra have been also included. There are a few methods permitting to calculate THD factors. An analytic method for the THD computation by the Cauchy method of residues has been presented in [1].

It is evident that in order to decrease the THD factor of the stepped function it is necessary to develop more precisely formed steps of the function. In NPC inverters it is impossible assuming that the PWM method is not considered but in cascaded inverters it requires to use a next $\mathrm{HB}$ inverter and a next supplying voltage $\boldsymbol{U}_{\boldsymbol{D} 3}$ up to numerous units [2].

The aim of this contribution is to prove that it is possible to diminish the THD factor in the cascaded inverter consisting of two HB inverters. Indispens able conditions to realize an inverter generating an optimal stepped voltage wave form $f_{N=6}$ are to be discussed in next chapters of the paper.

\section{Higher harmonics' content of the stepped wave form $f_{N=6}$}

Assuming that $f_{N=6}$ function is symmetrical in every halfinterval of the function period in relation to the symmetry axes $\boldsymbol{x}=\boldsymbol{\pi} / \mathbf{2}$ and $\boldsymbol{x}=\mathbf{3} \boldsymbol{\pi} / \mathbf{2}$, the shape of the $f_{N=6}$ may be defined by two parameters: step levels ratio $V_{0} / V_{\boldsymbol{l}}$ and the angle $\boldsymbol{\alpha}$ defining the "length" of the first step. For the wave form in Fig. 5 the ratio $\boldsymbol{V}_{\mathbf{0}} / \boldsymbol{V}_{\mathbf{1}}=\mathbf{0 , 5}$ and the angle $\alpha=\pi / 3$.

Two examples of function $\boldsymbol{F}_{\boldsymbol{N}=\mathbf{6}}$ approximation have been presented in Fig. 7 and 8. Parameters of wave form in Fig. 7 are the following: $\alpha_{0}=\pi / 6, \quad V_{0}=\mathbf{0 , 2 5 5 9}$, $\boldsymbol{V}_{\mathbf{1}}=\mathbf{0 , 8 2 7 0}$, so the ratio $\boldsymbol{\theta}=\boldsymbol{V}_{\boldsymbol{0}} / \boldsymbol{V}_{\boldsymbol{l}}$ is approximately equal to $\mathbf{0 , 3 1}$. The wave form spectrum is not so regular as presented before in Fig. 5 and 6. That wave forms have been built from $N$ regular and equal length pulses. Knowing number $N$ it is possible to define frequencies of all nonzero harmonics according to relation $(\boldsymbol{n} N \pm \mathbf{1}) \boldsymbol{f}_{\mathbf{1}}$, where $f_{l}$ denotes frequency of fundamental harmonic.

The wave in Fig. 7 has not the regular spectrum as spectra mentioned before. It includes lower harmonics including the third one but its THD factor doesn't exceed $\mathbf{2 1 , 6 2 \%}$ and the mean-square error $\boldsymbol{\delta}=\mathbf{0 , 0 2 2 2}$. This result seems to be very promising in comparison with $31,09 \%$ of the wave form in Fig. 5.

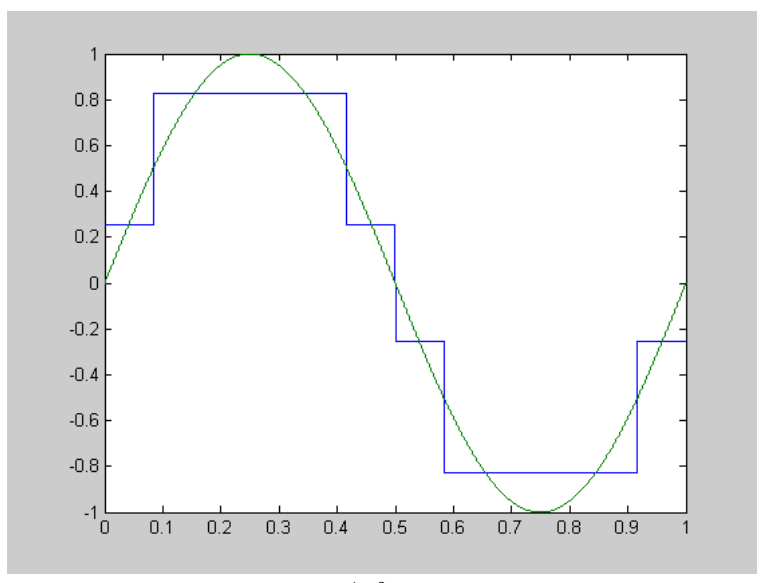

a) $f_{N=6}$

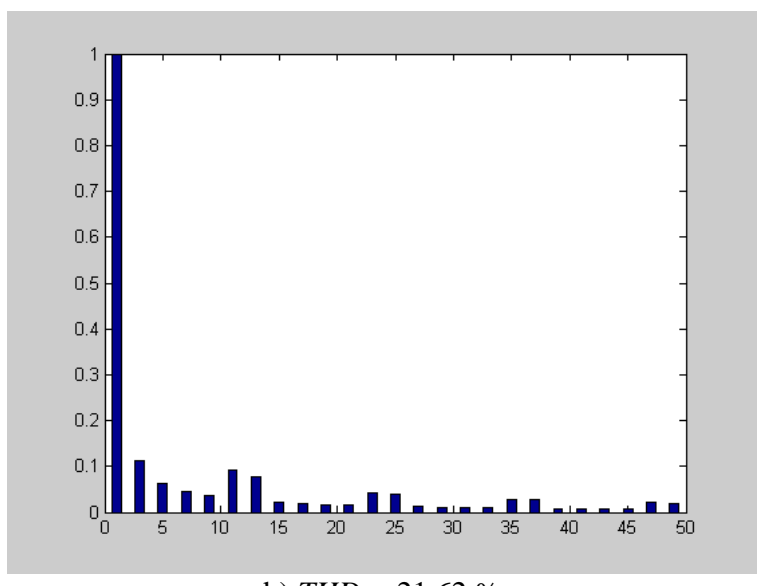

b) $T H D=21,62 \%$

Fig. 7. Approximation of $f(x)=\sin (x)$ function in the interval $<0,2 \pi)$ : a) wave form $f_{N=6}:(\alpha=\pi / 6, \theta=0,31$. $)$, b) harmonic spectrum. 
Even harmonics do not appear in wave forms considered in the paper. Odder harmonics $\boldsymbol{b}_{\boldsymbol{k}}$ appearing in stepped wave forms $\boldsymbol{f}_{N=\mathbf{6}}$ defined by parameters $\boldsymbol{V}_{\mathbf{0}}, \boldsymbol{V}_{\mathbf{1}}, \boldsymbol{\alpha}$ can be calculated according to expression:

$$
b_{k}=\frac{4}{k \pi}\left[V_{0}+\left(V_{1}-V_{0}\right) \cos (k \alpha)\right] \quad k=1,3,5, \ldots
$$

In which $\boldsymbol{V}_{\mathbf{0}}$ and $\boldsymbol{V}_{\mathbf{1}}$ denote step level values and the angle $\boldsymbol{\alpha}$ denotes the angle range of the first step.

The expression (1) is very useful and analysed largely in professionalliterature $[3,4]$. Thanks to $(1)$ it is possible to define parameters of the $f_{N=6}$ permitting to eliminate selected odder harmonics. There are many examples and discussions published in contributions concerning 3-level inverters. Assuming elimination of third and fifth harmonics it is possible to obtain a system of equations 2 .

$$
\left\{\begin{array}{c}
4\left[V_{0}+\left(V_{1}-V_{0}\right) \cos (\alpha)\right]=\pi \\
V_{0}+\left(V_{1}-V_{0}\right) \cos (3 \alpha)=0 \\
V_{0}+\left(V_{1}-V_{0}\right) \cos (5 \alpha)=0
\end{array}\right\}
$$

Solving this system we obtain the following parameters of the $f_{N=6}$ wave form:

Fig. 8. Approximation of $f(x)=\sin (x)$ function in the interval $<0,2 \pi)$ : a) wave form $f_{N=6}:(\alpha=\pi / 4, \theta=0,414)$, b) harmonic spectrum.

The formula (1) may be converted to the form

$$
b_{1}=\frac{4 V_{1}}{\pi}[\theta+(1-\theta) \cos (\alpha)]
$$

The fundamental harmonic $\boldsymbol{b}_{\boldsymbol{1}}$ is a function of two parameters: ratio of step levels $\boldsymbol{\theta}=\boldsymbol{V}_{\mathbf{0}} / \boldsymbol{V}_{\mathbf{1}}$ and angle range of first step

$$
b_{1}(\alpha, \theta)
$$

It is possible to present $\boldsymbol{b}_{\boldsymbol{1}}$ function in a three-dimensional rectangular coordinates system. The image of the function $\boldsymbol{b}_{\boldsymbol{I}}(\boldsymbol{\alpha}, \boldsymbol{\theta})$ has been presented in Fig.9. It was assumed that the range of parameters was limited to: $\alpha \in\left\langle 0,70^{\circ}\right\rangle, \theta \in\langle 0.1,1\rangle$ and $V_{l}=1$.

The amplitude of fundamental harmonic strongly diminishes for low values of $\boldsymbol{\theta}<\mathbf{0 , 3}$ and simultaneously increasing values of the angle $\boldsymbol{\alpha}>\mathbf{5 0}$. Then one can expect that THD factor increases because its value is $V_{0}=0,3927 ; \quad V_{1}=0,9481 ; \quad \alpha=\frac{\pi}{4} ; \quad b_{1}=1 ; \quad b_{3}=b_{5}=$ inversely proportional to the fundamental harmonic.

The final wave form is presented in Fig. 8. Note that the THD factor's value is $\mathbf{2 3 , 1 \%}$.

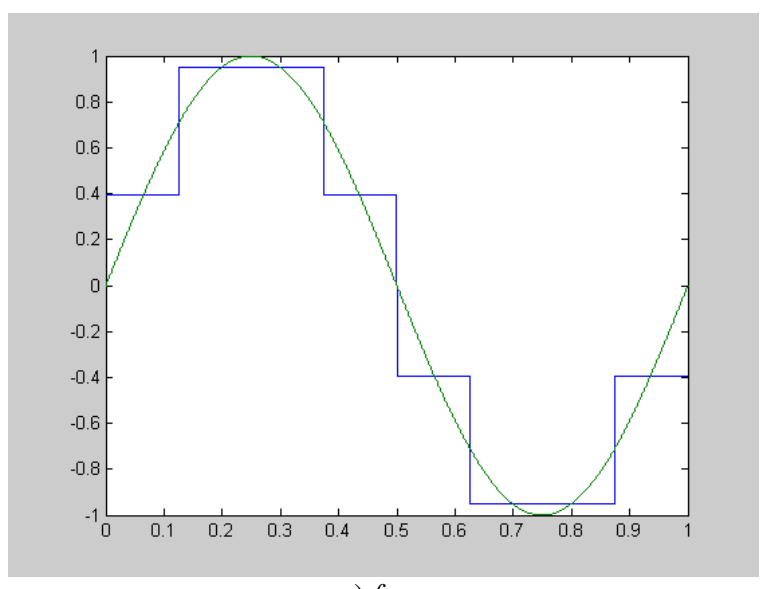

a) $f_{N=6}$

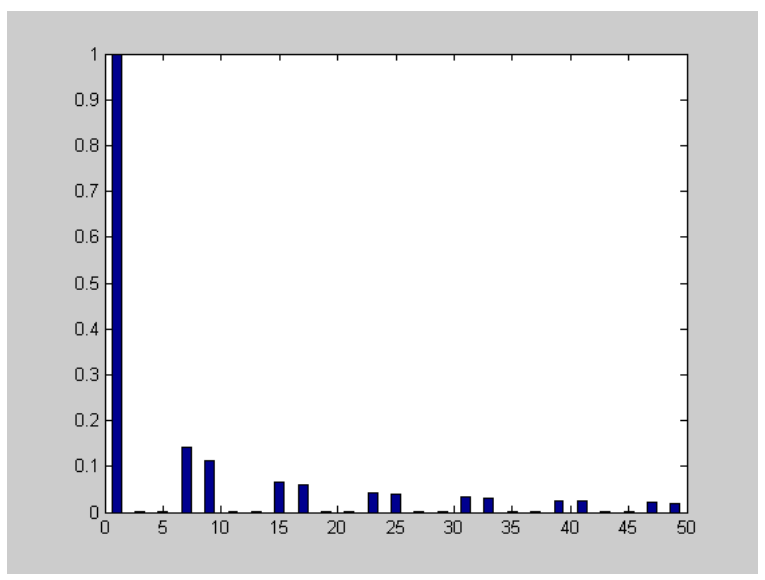

b) $T H D=23,1 \%$ b1 (alfa, theta)
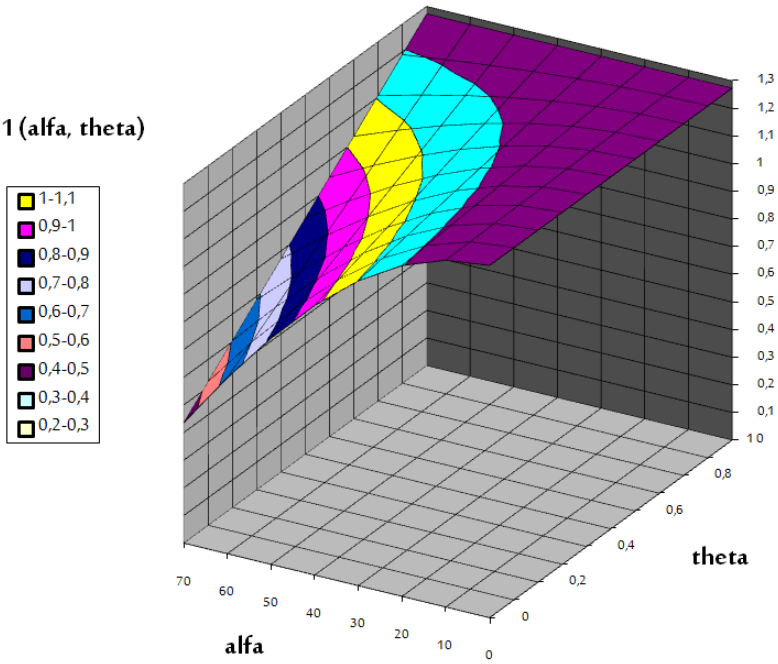

Fig. 9. The image of the function $b_{l}(\alpha, \theta)$ : Values of fundamental harmonic $b_{1}$ presented in 3-dimensional rectangular coordinates system: $\alpha \in\left\langle 0,70^{\circ}\right\rangle, \theta \in\langle 0.1,1\rangle$.

\section{Optimal shape of the wave form $f_{N=6}$}

Assuming the smallest THD factor value as a criterion of an optimal wave form $f_{N=6}$ shape it is possible to find relevant parameters $V_{0}, V_{l}, \alpha$ which assure the minimal value of this factor. According to the definition THD factor is given as:

$$
T H D=\frac{1}{b_{1}} \sqrt{\sum_{k=1}^{k=\infty} b_{2 k+1}^{2}}
$$


Taking into consideration (1) and introducing a function $\boldsymbol{f}(\boldsymbol{\alpha}, \boldsymbol{\theta})$ the THD definition may be expressed as:

$$
T H D=\frac{4 V_{1}}{\pi b_{1}} \sqrt{f(\alpha, \theta)}
$$

The $\boldsymbol{f}(\boldsymbol{\alpha}, \boldsymbol{\theta})$ is the function of two variables defined earlier: $\boldsymbol{\alpha}, \boldsymbol{\theta}$ and is given as:

$$
f(\alpha, \theta)=\sum_{k=1}^{k=\infty}\left\{\frac{1}{2 k+1}[\theta+(1-\theta) \cos [(2 k+1) \alpha]]\right\}^{2}
$$

The $\boldsymbol{f}(\boldsymbol{\alpha}, \boldsymbol{\theta})$ determines a wave form shape and in order to find a minimum of the THD factor it is obligatory to find a minimum of the function $f(\alpha, \theta)$. So, the question of THD minimum reduces to the question of $\boldsymbol{f}(\boldsymbol{\alpha}, \boldsymbol{\theta})$ minimum [5].

The domain of the function $\boldsymbol{f}(\boldsymbol{\alpha}, \boldsymbol{\theta})$ is determined by a plane area limited to a rectangle which dimensions are: $\mathbf{0}<\boldsymbol{\alpha}<\boldsymbol{\pi} / \mathbf{2}, \mathbf{0}<\boldsymbol{\theta}<\mathbf{1}$. Theoretically the variables $\boldsymbol{\alpha}$ and $\boldsymbol{\theta}$ could accept extreme values $\boldsymbol{\alpha}=\mathbf{0} \cup \boldsymbol{\alpha}=\boldsymbol{\pi} / \mathbf{2}$ a n d $\boldsymbol{\theta}=\mathbf{0} \cup \boldsymbol{\theta}=\mathbf{1}$ but practically they are limited to a definite range. The image of $\boldsymbol{f}(\boldsymbol{\alpha}, \boldsymbol{\theta})$ in a three-dimensional coordinate system has been presented in Fig. 10. The area corresponding to the lowest values has been denoted in blue colour.

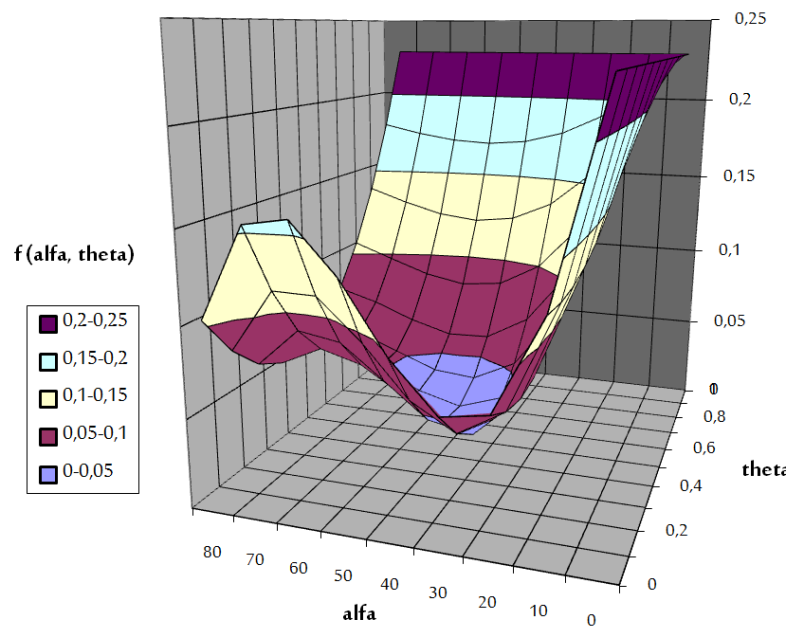

Fig. 10. Image of the function $f(\alpha, \theta)$ in three-dimensional coordinate system.

The next Fig. 11 presents a vertical projection of the function $f(\boldsymbol{\alpha}, \boldsymbol{\theta})$ on the $(\boldsymbol{\alpha}, \boldsymbol{\theta})$ plane. The blue area determines a set of optimal parameters according to the assumed criterion.

A few function $\boldsymbol{f}(\boldsymbol{\alpha}, \boldsymbol{\theta})$ diagrams for selected $\boldsymbol{\theta}$ have been presented in Fig. 12. The shape function $f(\alpha, \theta)$ reaches least values for two $\boldsymbol{\theta}$ coefficients: $\boldsymbol{\theta}=\mathbf{0 , 3}$ and $\boldsymbol{\theta}=\mathbf{0 , 4}$. Final analysis of calculation results ${ }^{1}$ proved that the function $f(\alpha, \theta)$ should have its expected minimum at point $(\boldsymbol{\alpha}=36, \boldsymbol{\theta}=\mathbf{0 , 3 5})$. The minimal value of the shape function is $f(\alpha, \theta) \approx 0.0325$.

${ }^{1}$ Calculations of $f(\alpha, \theta)$

have been done in Excel.

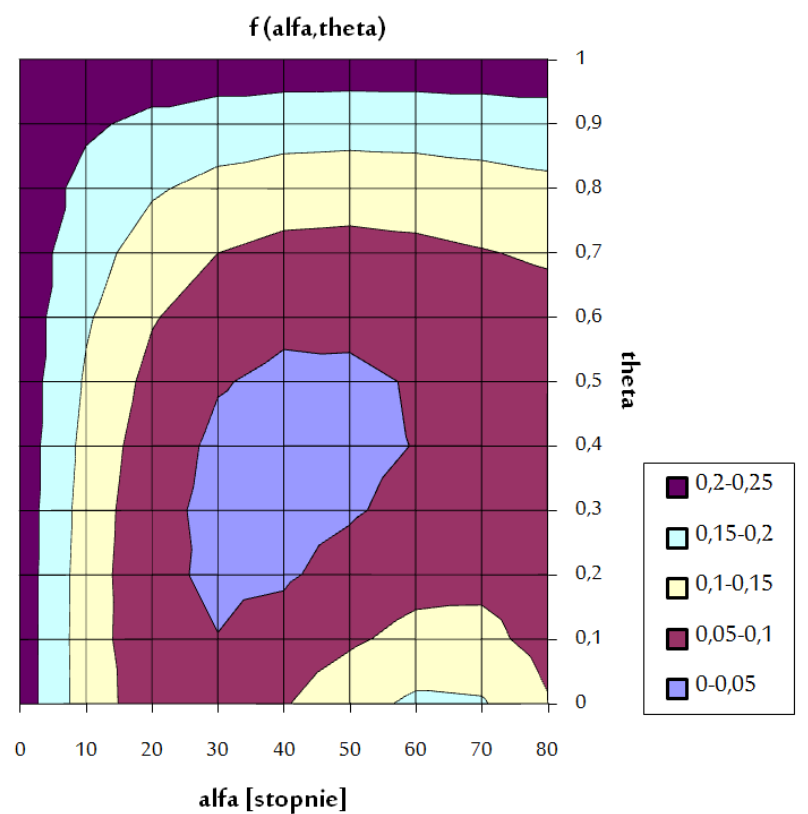

Fig. 11. Vertical projection of the function $f(\alpha, \theta)$ on $(\alpha, \theta)$ plane.

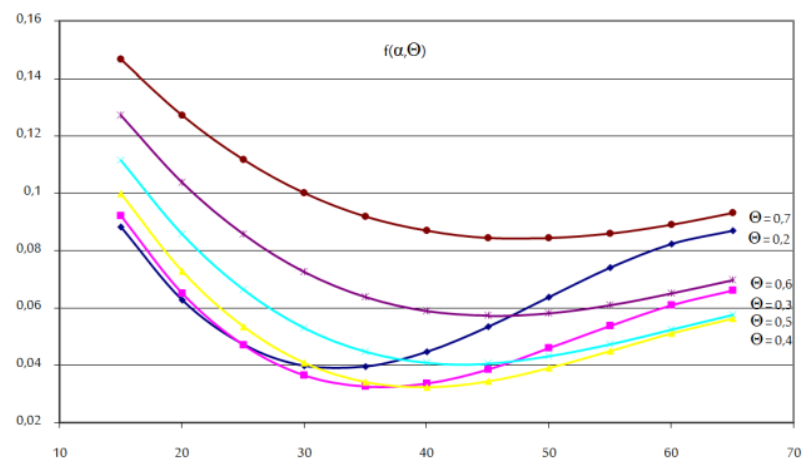

Fig. 12. The $f(\alpha, \theta)$ values for selected $\theta$ in the angle range $\left.\alpha<15^{\circ} 65^{\circ}\right\rangle$.

The wave form approaching to the best one according to aforementioned criterion as well as its spectrum have been presented in Fig. 13.

Parameters of the function $\boldsymbol{f}(\boldsymbol{\alpha}, \boldsymbol{\theta})$ are the following: $\boldsymbol{\alpha}=40^{\circ}, \boldsymbol{\theta}=\mathbf{0 , 4}$ and resulting in parameters of the $f_{N=6}$ are: $V_{0}=0,3655, V_{1}=0,9136$. The $T H D$ value is $20,98 \%$.

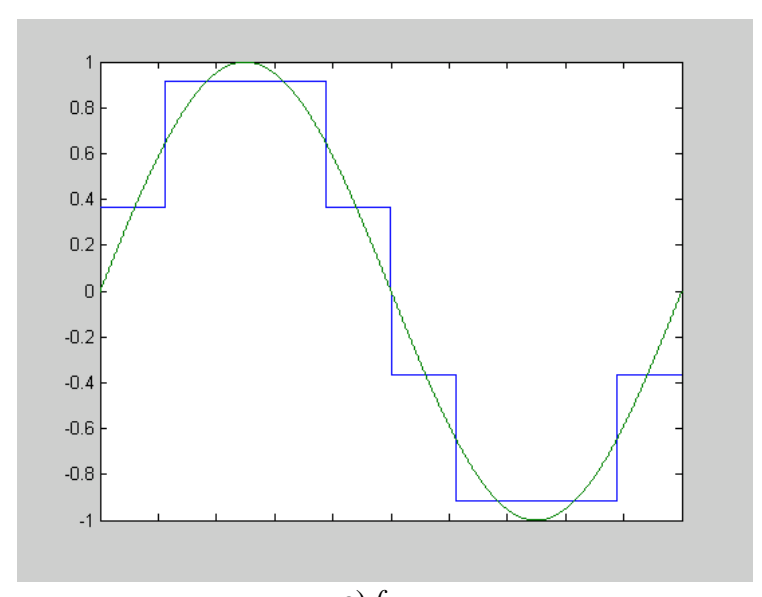

a) $f_{N=6}$ 


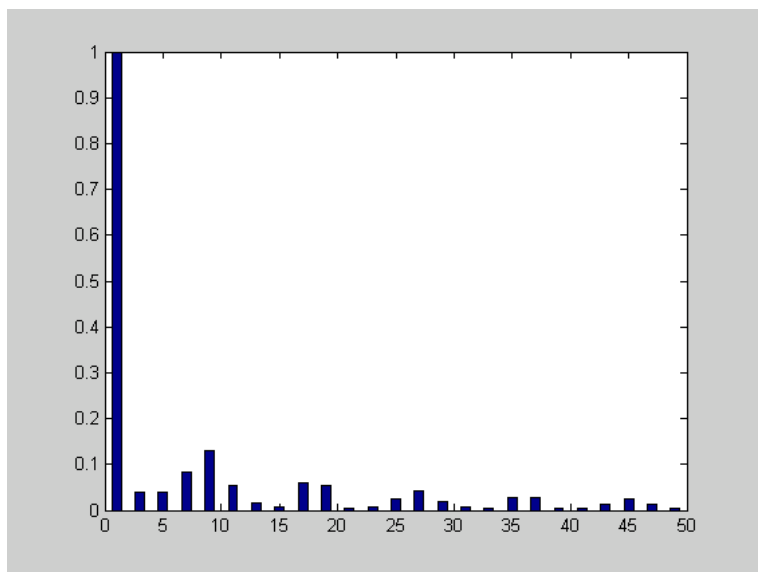

b) $T H D=20,98 \%$

Fig. 13. Approximation of the function $f(x)=\sin (x)$ in the interval $<0,2 \pi$ )by use of the stepped wave form $f_{N=6}(\alpha=2 \pi / 9, \theta=0.4):$ a) $f_{N=6}$, b) spectrum.

\section{Cascaded 3-level inverter with reduced THD}

A schematic diagram of a cascaded inverter has been presented in Fig. 14. In such a kind of the 3-level cascaded VSI the summing block is not needed because output voltages of constituent inverters are connected in series. So, the final output voltage is created as a result of a sum or a difference of two voltage values.

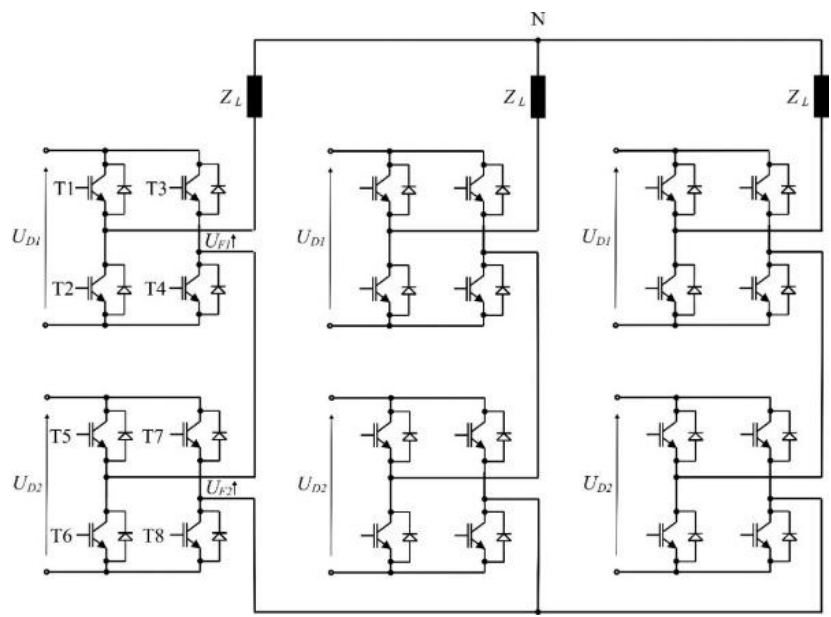

Fig. 14. Cascaded three phase 3 -level inverter.

Applying a very simple rule of control it is possible to obtain three-phase 3-level voltage wave form supplying the output load. For illustration the switching sequence: $T 1-T 4, T 1-T 4 \cap T 5-T 8, T 1-T 4, T 2-T 3, T 2$ $T 3 \cap T$ 6 - T 7, T $2-T 3$ presented in Chapter I assures 3level wave forms. It is evident that successive phase control signals are adequately shifted. According to results of wave form optimization a very good approximation is possible to realize if angle range and supply voltages have to satisfy parameters: $\alpha=40^{\circ}, \quad \theta=0,4, \quad V_{0}=0,3655$, $V_{1}=0,9136$. Resulting in supply voltages $\boldsymbol{U}_{\boldsymbol{D} \boldsymbol{1}}$ and $\boldsymbol{U}_{\boldsymbol{D} 2}$ have to be calculated to reach an assumed value of the fundamental harmonic. For instance the inverter supplied by $\boldsymbol{U}_{D 1}=110 \mathrm{~V}$ and $\boldsymbol{U}_{D 2}=165 \mathrm{~V}$ generates stepped wave forms $\boldsymbol{f}_{N=6}$ that the fundamental harmonic $\boldsymbol{b}_{1}=300 \mathrm{~V}$ and $T H D=20,98 \%$.

\section{Conclusion}

According to assumed criterion of minimal THDthe best 3-level stepped voltage can be realized if both parts of a standard 3-level NPC inverter or both $\mathrm{H}$ inverters in cascaded topology are supplied by two different DC voltages.Research results presented in the paper prove that in orderto obtain minimal THD factor of the output voltage it is necessary to use precisely calculated parameters of DC voltage supplying the inverter units. Next requirement relates to parameters of the stepped

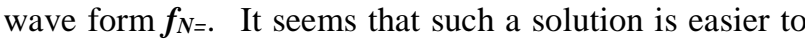
develop in cascaded inverters than in 3-level ones. Optimal parameters of the output voltage: $\alpha=36^{\circ}(\pi / 5)$, $\boldsymbol{\theta}=\mathbf{0 , 3 5}$ guarantee the minimal value of the THD factor. Theoretically the same result could be achieved in standard NPC 3-level inverters but it would be necessary to divide intermediary DC voltage according to optimal parameters as well as to maintain this state during active work of the inverter.

\section{References}

[1] I. V. Blagouchine, E. Moreau, "Analytic Method for the Computation of the Total Harmonic Distortion by the Cauchy Method of Residues", IEEE Transactions on Communications, 2011, VOL. 59, no. 9.

[2] N. Suresh, S. R. Babu, Reduction of Total Harmonic Distortion in Cascaded H-Bridge Inverter by Pattern Search Technique, IEEE Transactions on Communications, 2011, VOL. 59, no. 9.

[3] J. Rodriguez, J.-S. Lai, F. Z. Peng, Multilevel Inverters: A Survey of Topologies, Controls and Applications. IEEE Transactions on Industrial Electronics, 2002, Vol. 49, no. 4, pp. 724-739.

[4] L. M. Tolbert, J. Chiasson, K. McKenzie, Zhong Du, Elimination of harmonics in a multilevel converter with non equal DC sources, Applied Power Electronics Conference and Exposition, 2003. APEC '03. Eighteenth Annual IEEE, 2003, vol. 9-13, pp. 589 - 595.

[5] Iwaszkiewicz J.: Mathematical Models of Power Electronics Multilevel Converters - Analysis and Applications, Proceedings of Electrotechnical Institute 227'6, Warsaw, Poland, March 2006, p. 142. 